\title{
A period study of the W UMa type contact binary V 781 Tauri
}

\author{
Qingyao Liu and Yulan Yang \\ Yunnan Observatory, Chinese Academy of Sciences, Kunming, Yunnan, China \\ United Laboratory of Optical Astronomy, Chinese Academy of Sciences, China \\ National Astronomical Observatories, Chinese Academy of Sciences, China \\ e-mail: bily@public.km.yn.cn
}

Received May 25; accepted November 2, 1999

\begin{abstract}
New times of minimum light determined from the observations of the W UMa type contact binary V 781 Tau are presented. According to these times of minima and those collected from the references, the change in the orbital period of the system is analyzed. The result reveals that the orbital period of V 781 Tau decreased continually from 1949 to 1998 and the change rate in the period is $\delta p / p=-5.010^{-11}$. The decrease in the period of the binary is explained by a model of the contracting secondary component of the system. The shrinking velocity of $6.7710^{-5} \mathrm{~cm} \mathrm{~s}^{-1}$ as responsible for the change in the orbital period of the system is obtained, which is in agreement with the results shown in the study of the thermal relaxation oscillation of contact binaries by Wang (1994).
\end{abstract}

Key words: stars: binaries: close — stars: binaries: general — stars: kinematics

\section{Introduction}

One of many interesting problems in the study of W UMa contact binaries is to explain the change in the orbital period of the systems. The variety of the period changes of W UMa binaries makes it difficult to understand their real physical mechanism. However, it is clear that the change in the period of a W UMa contact binary should result from their unstable structure and evolution. According to Lucy (1976), Flannery (1976) and Robertson \& Eggleton (1977), a zero-age W UMa binary is obliged to undergo periodic thermal relaxation oscillation about a state of marginal contact. It can be expected that some observable changes in the orbital period of the systems should arise because of such oscillations. Unfortunately, the time scale of $10^{7}$ years of the oscillations is too long to be observed completely for the short astronomical history, but it

Send offprint requests to: Qingyao Liu is noticed that the results obtained by Wang (1994) could provide a way to do something for this test. Wang's study suggests that the two subtypes of W UMa type binaries are in two different thermal relaxation oscillation states: the secondary components of W-subtypes of W UMa type binaries are shrinking whereas the ones of A-subtypes are swelling. Therefore, the orbital period of the W-subtypes should be continually decreasing whereas that of the A-subtypes should be continually increasing. For acquiring the observational evidence to test the results of Wang's study (1994) the authors are interested in the observation and analysis of the change in the period of a W UMa type binary. The W UMa type binary V 781 Tau is the first of our program stars.

The variability of V 781 Tau was discovered by Harris (1979), who classed it as a new W UMa type binary with the orbital period of 0 d 3994 . Berthold (1981) derived 14 times of minimum light of the system from 232 photographic plates taken from 1958 to 1977 and showed an orbital period with a sudden increase in 1.5 seconds in 1971. After two years he presented a revision of his previous results (Berthold 1983). The photoelectric times of minimum light of V 781 Tau were published by Diethelm (1981a, 1981b), Pohl (1987) and Keskin (1989). Cereda et al. (1988) made extensive photoelectric observations in $B$ and $V$ bands and published very good light curves. They found a photometric solution of their light curves and obtained rough parameters of the binary. The observations of the radial velocity and absolute dimensions were published by Lu (1993), who re-analyzed the Cereda et al.'s light curves by using the Wilson-Devinney code, with the results revealing that V 781 Tau is a W-subtype W UMa binary and the primary component resembles a main-sequence star and the secondary is very close to the ZAMS in the HR diagram and in the mass-radius diagram. 
Table 1. The coordinates of the comparison and check stars

\begin{tabular}{lcc}
\hline star & $\begin{array}{c}\text { R.A. } \\
(1998.0)\end{array}$ & $\begin{array}{c}\text { Dec. } \\
(1998.0)\end{array}$ \\
\hline V 781 Tau & $05^{\mathrm{h}} 50^{\mathrm{m}} 06^{\mathrm{s}}$ & $26^{\circ} 57^{\prime} 41^{\prime \prime}$ \\
comparison 1 & 055015 & 265947 \\
comparison 2 & 055018 & 265642 \\
check & 055013 & 265634 \\
\hline
\end{tabular}

\section{Observation}

The observations of $\mathrm{V} 781$ Tau in $B$ and $V$ bands were carried out on December 31, 1997 and on January 4, 1998 with the PI1024TKB CCD photometric system attached to the $100-\mathrm{cm}$ reflector telescope at the Yunnan Observatory in China. The effective field of view of the photometric system is 6.5 square arc minute at the Cassegrain focus and its $B V$ color system (Yang \& $\mathrm{Li}$ 1999) approximates the standard Johnson $B V$ photometric system. The three brighter stars except for the variable in the CCD field are used as the comparison and check stars, which are different from those used by other observers, so their coordinates together with those of the variable are given in Table 1. Integration time for each image is 100 seconds. In each of the two bands, 45 images during the primary eclipse and 38 images during the secondary eclipse have been obtained to determine times of minima of the system.

The photometric program of the IRAF software is used to reduce the images. The results of the reductions show that the difference between the magnitudes of the check star and the comparison star 1 is constant within probable errors of $\pm 0.008 \mathrm{mag}$ for the $V$ band and $\pm 0.011 \mathrm{mag}$ for the $B$ band. However, the difference between the magnitudes of the check star and the comparison star 2 is $\pm 0.018 \mathrm{mag}$ for the $V$ band and $\pm 0.022 \mathrm{mag}$ for the $B$ band. So the star 1 is used as a comparison star to reduce our observations of V 781 Tau. Extinction corrections are not made because the comparison star 1 is very close to the variable.

From the observation made during the primary and the secondary eclipses, a mean epoch of the primary minimum light in the $B$ and $V$ bands and that of the secondary minimum light in the $B$ and $V$ bands are derived by means of the parabola fitting. The present times of minima together with those collected from the references are listed in Table 2, where the second column entry denotes the observation method: PG refers to the photographic observations and PHE to the photoelectric one.

\section{Change in the period and explanation}

The $(\mathrm{O}-\mathrm{C})_{1}$ residuals in Table 2 are calculated by using the ephemeris given by Cereda et al. (1988), i.e.

Min.I = HJD 2443853.9096+0.3449094E.

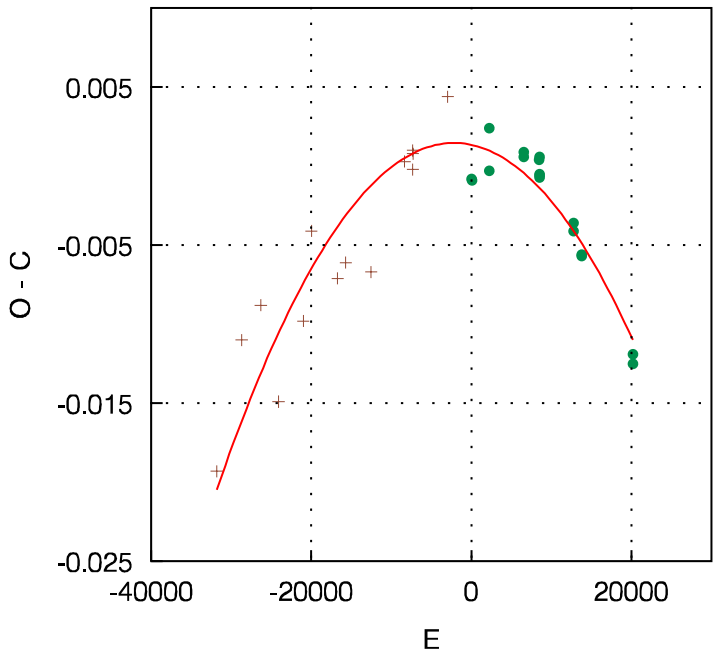

Fig. 1. The O-C diagram of the period change for V 781 Tau. The solid circles indicate the photoelectric observations and the crosses express the photographic one

The $(\mathrm{O}-\mathrm{C})$ diagram of the change in the orbital period of $\mathrm{V} 781$ Tau is shown in Fig. 1, where $(\mathrm{O}-\mathrm{C})_{1}$ residuals listed in Table 2 are used. It is clear that the period of this binary star decreased continually from 1949 to 1998 . All epochs in Table 2 are used for a least square solution to obtain a quadratic ephemeris. The improved ephemeris is described as follows:

$$
\begin{aligned}
\text { Min.I }= & \text { HJD } 2443853.9110(15)+0.34490929(18) E \\
& -2.5(2) 10^{-11} E^{2}
\end{aligned}
$$

which is used to calculate the $(\mathrm{O}-\mathrm{C})_{2}$ in Table 2. The period change rate of V781 Tau is $\delta p / p=-5.010^{-11}$.

The secular instability of the secondary component of a W UMa type binary arises because they obtain the luminosity transferred from the primary component (Lucy \& Wilson 1979; Hazlehurst 1985; Wang 1994). So it could be suggested that decrease in the period of V 781 Tau is caused by the contraction of the secondary component, then its shrinking velocity can be calculated from the decrease rate in the orbital period.

The Kepler's third law can be written as

$A^{3}=74.5 p^{2} M$

where $A$ is the separation between the two components in solar radii, $p$ represents the orbital period in days and $M$ indicates the total mass of the two components in solar mass. From the definition of the relative radius of one of the two components, one may have

$A=\frac{R_{1}+R_{2}}{r_{1}+r_{2}}$.

According to Binnendijk (1970) and Lacy (1977), one may have

$r_{1}+r_{2}=0.76$ 
Table 2. Times of minimum light for V 781 Tau

\begin{tabular}{lccccc}
\hline JD(hel.) & Meth. & $E$ & $(\mathrm{O}-\mathrm{C})_{1}$ & $(\mathrm{O}-\mathrm{C})_{2}$ & Ref. \\
$2400000+$ & & & & & \\
\hline 32881.460 & PG & -31812.5 & -0.0193 & 0.001 & Berthold (1983) \\
33950.515 & PG & -28713.0 & -0.0110 & 0.005 & ditto \\
34775.368 & PG & -26321.5 & -0.0088 & 0.004 & ditto \\
35540.371 & PG & -24103.5 & -0.0149 & -0.004 & ditto \\
36610.285 & PG & -21001.5 & -0.0098 & -0.003 & ditto \\
36957.442 & PG & -19995.0 & -0.0041 & 0.002 & ditto \\
38088.397 & PG & -16716.0 & -0.0071 & -0.003 & ditto \\
38440.378 & PG & -15695.5 & -0.0061 & -0.003 & ditto \\
39536.327 & PG & -12518.0 & -0.0067 & -0.006 & ditto \\
40981.332 & PG & -8328.5 & 0.0003 & -0.000 & ditto \\
41329.345 & PG & -7319.5 & -0.0002 & -0.001 & ditto \\
41330.381 & PG & -7316.5 & 0.0010 & 0.000 & ditto \\
41337.279 & PG & -7296.5 & 0.0008 & -0.000 & ditto \\
42839.363 & PG & -2941.5 & 0.0044 & 0.003 & ditto \\
43853.9088 & PHE & 0 & -0.0008 & -0.0022 & Cereda et al. (1988) \\
43874.9482 & PHE & 61.0 & -0.0009 & -0.0023 & ditto \\
44636.339 & PHE & 2268.5 & 0.0024 & 0.0014 & ditto \\
44637.371 & PHE & 2271.5 & -0.0003 & -0.0013 & ditto \\
46113.4117 & PHE & 6551. & 0.0006 & 0.0010 & Pohl et al. (1987) \\
46115.309 & PHE & 6556.5 & 0.0009 & 0.0013 & ditto \\
46775.4651 & PHE & 8470.5 & 0.0004 & 0.0018 & Cereda et al. (1988) \\
46788.3981 & PHE & 8508.0 & -0.0007 & 0.0007 & ditto \\
46798.4005 & PHE & 8537.0 & -0.0006 & 0.0007 & ditto \\
46802.3682 & PHE & 8548.5 & 0.0006 & 0.0020 & ditto \\
46802.5396 & PHE & 8549.0 & -0.0005 & 0.0009 & ditto \\
48268.2285 & PHE & 12798.5 & -0.0041 & 0.0000 & Keskin et al. (1989) \\
48268.4014 & PHE & 12799.0 & -0.0036 & 0.0005 & ditto \\
48607.2728 & PHE & 13781.5 & -0.0057 & -0.0008 & ditto \\
48607.4454 & PHE & 13782.0 & -0.0056 & -0.0007 & ditto \\
50814.1688 & PHE & 20180.0 & -0.0125 & -0.0015 & this paper \\
50818.1359 & PHE & 20191.5 & -0.0119 & -0.0008 & this paper \\
\hline & & & & &
\end{tabular}

and

$\frac{R_{2}}{R_{1}}=q^{0.92}$.

Inserting the Eqs. (4), (3) and (2) into the Eq. (1), one may acquire

$R_{2}^{3}\left(1+\frac{1}{q^{0.92}}\right)^{3}=32.7 p^{2} M$.

Assuming conservation of total mass of the system, from the Eq. (5), one can obtain

$\frac{\mathrm{d} R_{2}}{\mathrm{~d} t}=\frac{21.8 p M q^{2.76}}{R_{2}^{2}\left(1+q^{0.92}\right)^{3}} \frac{\mathrm{d} p}{\mathrm{~d} t}$.

Since the period of V 781 Tau decreases, from the Eq. (6) and the parameters published by $\mathrm{Lu}$ (1993), one may find a contracting velocity of the secondary component, $\mathrm{d} R_{2} / \mathrm{d} t=-6.7710^{-5} \mathrm{~cm} \mathrm{~s}^{-1}$, namely the contraction of $21.4 \mathrm{~m} /$ year, which is in agreement with the results shown in the study of the thermal relaxation oscillation of contact binaries by Wang (1994).

\section{Discussion}

The decrease in the orbital period of V 781 Tau has presented the evidence to test the interesting results presented by Wang (1994), who suggested that the two subtypes of W UMa binaries should be in two different thermal relaxation oscillation states: the secondary components of the W-subtypes of W UMa type binaries are shrinking whereas the ones of A-subtypes are swelling. According to Wang (1994), a W-subtype W UMa binary has a contracting secondary component, which releases some gravitational energy to support its surface effective temperature higher than that of the primary component. The luminosity radiated by the secondary component consists of the three parts, the first is from the nuclear reaction in the secondary, the second is from the transferred luminosity from the primary and the third is from the released gravitational luminosity by the secondary component. Suppose the secondary component with a mass of $m_{2}$ and a radius of $R_{2}$ spherically symmetric, then its gravitational energy $E_{\mathrm{g}}$ is written (Kippenhahn 
\& Weigert 1990) as follows:

$E_{\mathrm{g}}=\frac{3 G m_{2}^{2}}{(n-5) R_{2}}$

where $n=3$ for the main-sequence stars. For the low mass secondary components of W-subtype W UMa binaries, $n=1.5$ adopted by Wang (1994). From the Eq. (7), the following equation can be derived

$\frac{\mathrm{d} E_{\mathrm{g}}}{\mathrm{d} t}=\frac{3 G m_{2}^{2}}{(5-n) R_{2}^{2}} \frac{\mathrm{d} R_{2}}{\mathrm{~d} t}$.

$\mathrm{Lu}$ (1993) gave the physical parameters of V 781 Tau and pointed out that the components of this system are main-sequence stars. A luminosity generated from the nuclear reactions of the secondary component with a mass of $0.501 m_{\odot}$ is about $0.048 L_{\odot}$. Assuming that the transferred luminosity from the primary component could only make the surface effective temperature of the secondary component the same as that of the primary component, then a maximum value of the transferred luminosity was about $0.65 L_{\odot}$. The observed luminosity of the secondary component is $0.71 L_{\odot}$ and it can be supposed that the over-luminosity of $0.012 L_{\odot}$ was the gravitational luminosity released by the secondary component. From the Eq. (8), one may find a minimum contracting velocity of the secondary component, $\mathrm{d} R_{2} / \mathrm{d} t=-2.4510^{-6} \mathrm{~cm} \mathrm{~s}^{-1}$. This minimum shrinking velocity is less than the contracting velocity of $6.7710^{-5} \mathrm{~cm} \mathrm{~s}^{-1}$ calculated from the decrease in the period, because the superior limit of the transferred luminosity from the primary component is adopted. In fact, adopting the contracting velocity of $6.7710^{-5} \mathrm{~cm} \mathrm{~s}^{-1}$ calculated from the decrease in the period and from the Eq. (8), one may find the gravitational luminosity released by the secondary component, $\mathrm{d} E_{\mathrm{g}} / \mathrm{d} t=0.330 L_{\odot}$. Thus, the transferred luminosity from the primary component is only about $0.33 L_{\odot}$ rather than $0.65 L_{\odot}$.

The paradox of the over-luminous secondary component of the W UMa binary was explained by the introduction of large-scale energy transfer from the primary to the secondary (Lucy 1968). In the present discussion for $\mathrm{V} 781 \mathrm{Tau}$, the secondary component of the system is continuously shrinking at the velocity of $6.7710^{-5} \mathrm{~cm} \mathrm{~s}^{-1}$ and releasing the gravitational luminosity of $0.330 L_{\odot}$, while the orbital period of the system was continuously decreasing with the change ratio of $\delta p / p=-5.010^{-11}$. Then the transferred luminosity from the primary component may be only a part of the over-luminosity, the other part may be from the gravitational luminosity released by the secondary component when it shrinks. According to Mochnacki (1981), the ratio of energy transfer of V 781 Tau should be of $\Delta L / L_{1}=0.45$, but in the present discussion, that is only of $\Delta L / L_{1}=0.22$.

It is interesting to notice that the $\mathrm{W}$-phenomena of W UMa binaries arise also because of the contracting secondary components rather than only because the energy transfer from the primary to the secondary. The transferred luminosity from the primary should never cause the temperature of the secondary component to be higher than that of the primary if there were non-gravitational luminosity released by the secondary component.

In order to test the contracting model of the secondary components of the W-subtype W UMa Binaries, observations and analyses in the orbital period of more systems will be needed.

Acknowledgements. The authors would like to thank Dr. F. Li for his assistance in the observation and Dr. P.G. Niarchos for his useful advice. The authors would also like to express their gratitude for the support from the Chinese National Science Foundation Committee and the Chinese Academy of Sciences.

\section{References}

Berthold T., 1981, IBVS No. 1942

Berthold T., 1983, IBVS No. 2443

Binnendijk L., 1970, Vistas Atron. 12, 217

Cereda L., Misto A., Niarchos P.G., Poretti E., 1988, A\&AS 76,255

Diethelm R., 1981a, Bed. Beo. Schweizer. Astron. Ges. 52, 7 Diethelm R., 1981b, Bed. Beo. Schweizer. Astron. Ges. 53, 4 Flannery B.P., 1976, ApJ 205, 317

Harris A.W., 1979, IBVS No. 1556

Hazlehurst J., 1985, A\&A 145, 25

Keskin V., Pohl E., 1989, IBVS No. 3355

Kippenhahn R., Weigert A., 1990, Stellar Structure and Evolution. Berlin Springer, p. 184-185

Lacy C.H., 1977, ApJS 34, 479

Lu W.X., 1993, AJ 105, 646

Lucy L.B., 1968, ApJ 151, 1123

Lucy L.B., 1976, ApJ 205, 208

Lucy L.B., Wilson R.E., 1979, ApJ 231, 502

Mochnacki R., 1981, ApJ 245, 650

Pohl E., Akan M.C., Ibanoglu C., Sezer C., Gudur N., 1987, IBVS No. 3078

Robertson J.A., Eggleton P.P., 1977, MNRAS 179, 359

Wang J.M., 1994, ApJ 434, 277

Yang Y.L., Li L.F., 1999, Publication of Yunnan Observatory, No. 1, p. 32 